\title{
influencia del polvo del horno de cemento sobre plantas y animales
}

F. PAJENK AMP

(Zement-Kalk-Gips», 50, núm. 3, marzo 1961, pág. 88

\begin{abstract}
Afnopsis Fl pretendido wpolvo de cementos de los alrededores de las fábricas no es de cemento sino que se compone, en sa mayor parte, de caliza molida muy fina. Es, por tanto, un producto intermedio de la fabricación del cemento. Sería mucho más correcto llamarle «polvo del harno de cementow.

Continuamente alegan los agricultores que este "polvo del horno de cementon es dañino para plantas y animales, $y$ en los úlimos años, para probar la validez de tales asertos, se han levado a cabo numerosas investigaciones científicas para estudiar los efectos de este polvo.

Estamos hoy día en posesión de una serie de trabajos muy completos que dan una idea clara sobre el efecto del polvo del horno de cemento. Los animales domésticos útiles (por ejemplo: corderos, vacas de leche) no sufren ningún perjuicio clînico, ninguna deficiencia de su estado general, a pesar de serle administradas grandes dosis de polvo.

Los ensayos agrícolas con plantas útiles (avena, trébol, remolacha azucarera, pastos) que han sido efectuados durante varies años, han demostrado que ni siquiera cantidades elevadas de polvo provoean ni perjuicios ni influencias nefastas sobre el crecimiento y sobre If recoleceion.
\end{abstract}

En conexión con la expulsión de polvo de las fábricas de cemento, continuamente se ha escrito y se ha hablado sobre el «polvo de cemento».

Sin embargo, el polvo, que es expulsado al exterior junto con los gases de escape del homo de fabricación de clínker, consiste, esencialmente, en piedras calizas parcialmente molidas y desacidificadas. Es una especie de producto intermedio en la fabricación del cemento, y de aquí que la industria de cemento esté empeñada, por razones puramente egoístas, en reducir al mínimo esta expulsión de polvo. Se debería, por tanto, indicar continuamente, y de modo especial por medio de toda clase de publicaciones, que esta cuestión del polvo de la industria del cemento son partículas de piedra caliza molida y desacidificada, pero nunca «polvo de cemento». Proponemos, para su utilización futura, la calificación de este polvo mediante el término: «Polvo del horno de cemento».

Estamos hoy en condiciones de desempolvar en un 100 por 100 toda la maquinaria que se utiliza en la molturación de clínker, en el envasado del cemento y en su transporte, de tal manera que el «auténtico polvo de cemento» no salga (y se pierda) al exterior. Desgraciadamente, no nos es posible hacer salir libres de polvo los gases de escape del horno que se producen en la cocción del cínker, así es que tenemos que contar, aunque en proporciones pequeñísimas, con la expulsión de polvo del horno.

Por parte de los agricuitores se alegan constantemente argumentos sobre las influencias dañinas de este «polvo del horno de cemento» en las plantas y animales. Para contrarrestar esta serie de afirmaciones se han llevado a cabo, en los últimos años, una serie de investigaciones.

La tabla núm. 1 nos da una idea sobre alguno de los importantes trabajos realizados sobre la inHuencia del polvo del horno de cemento en plantas y animales. En esta misma tabla están también incluidos trabajos que tratan de la infuencia de ese polvo sobre los hombres. Aunque propiamente no pertenece a este tema, debe señalarse una cietta semejanza entre el daño del polvo del horno de cemento y las investigaciones realizadas a este respecto sobre el organismo animal. 
TABLA 1. Trabajos sobre la influencia del polvo del horno de cemento.

\section{SOBRE LOS HOMBRES}

F. Schotr: Las influencias del polvo de cemento sobre los pulmones y el problema de la tuberculosis en el trabajador de la industria cementera.

Referencia: «Zementverlag Gmbh.», Charlottemburg, 1926.

Las investigaciones médicas realizadas sobre trabajadores en los distintos escalones de la producción, en el ámbito de las instalaciones de cemento, no demostraron ninguna influencia, sobre la salud de las personas exploradas, del polvo aspirado (tanto polvo de cemento como polvo del horno de cemento). (La expulsión de polvo de las fábricas de cemento en el año 1926 era fundamentalmente mayor que hoy.)

R. Fimiani y N. Castellino: Las condiciones higiénicas en ma fábrica de cenento.

Referencia: Fol. med., núm. 12, p. 1298-1325, diciembre 1958.— «Bull. Doc. bibliogr.\$, 10 (1959), núm. 4, p. 56 Investigaciones sobre 227 trabajadores de la industria del cemento en las diversas secciones de la producción, con simultánea determinación en cada caso del polvo contenído en el aire. No se aprecia ningún incremento de enfermedades a causa de la influencia de polvo. Ninguna enfermedad que, con seguridad, pueda ser atribujda a la influencia del cemento o del polvo del horno de cemento.

N. Mongeti-Scinnameo: Sobre la patologia del polvo de cemento. Observaciones clínicas radiologicas sobre 350 trabajadores de la industria del cemento.

Referencia: Fol. med, núm. 5, p. 469-507, mayo 1959.—*Bull. Doc. bibliogr.», 10 (1959), núm. 6, p. 36.

El diagnóstico no dio silioosis alguna, ni tuberoulosis activa, ni se sobrepasó la proporción normal de casos de tuberculosis enquistada.

E. GrandEAN : Investigaciones sobre la influencia del polvo del horno de cemento, y las impurificaciones del aire exterior sobre la salud de la población próxima.

Separata: El problema del polvo de la industria del cemento, del informe núm. 47 del año 1957 , y del informe núm. 49 dệ año 1959, de la Unión Suiza de Fabricantes de Cemento-Cal-Yeso.

Influencia del polvo del horno de cemento sobre la población vecina. Estudios estadísticos sobre 311 asegurados en poblaciones con fábricas de cemento (Hoiderbank, Wildegg), y 631 asegurados en lugares sin fábricas de cemento (Ruperswil, Hunzenschwil, Schafischeim, Niederlenz). Los estudios celebrados en los años de 1952 a 1956, no han resaltado ningún incremento de enfermedades en las poblaciones domiciliadas en Wildegg Holderbank como consecuencia de la emisión de polvo en relación con las poblaciones de los otros lugares.

\section{SOBRE LOS ANTMALES}

P. Cohrs y G. Trautwein: Investigaciones experimentales sobre la influencia del polvo de cemento sobre los animales. Referencia: Archivo para Medicina Experimental Veterinaria, Bd., XIII (59), p. 403-421.

Investigaciones sobre dos bueyes, 17 , corderos y 16 conejos que han digerido y aspirado polvo de horno de cemento (ver texto).

E. Scrutumann: Ensayos encargados por el Ministerio Federal de Agricultura y de Repoblación Forestal. (Todavia no publicados.)

Pulverización de plantas alìmenticias con polvo del horno de cemento y alimentación, en grandes cantidades, de ganado vacuno. Sir resultado positivo.

\section{SOBRE LAS PLANTAS}

H. Stratmann y H. van Haut : Ensayos sobre vegetales con aire cargado de polvo de cemento. Investigación encargada por la Unión de Fabricantes de Cemento Alemanes. (Aún no publicados.)

Resulfados de las investigaciones de los años de 1954 a 1956, principalmente en ensayos de laboratorio. (Ver texto.) 
H. Fontmans: Aportación a la cuestión de la influencia del polvo de las fábricas de cemento sobre las plantas y el suelo. Tesis elaborada en colaboración con la Escuela de Fomento de la Explotación Agrícola, Bochum (Dr. Bergerhoff).

Referencia: Publicación agricola de Gmbh. H. Hiltrup Westfalen, 1957.

Observaciones que duráron varios años, sobre el especial comportamiento de cultivos jóvenes de invernadero cuyas ventanas (del invernadero de primavera) han sido cubiertas con cantidades variables de polvo del horno de cemento. (Ver texto.)

H. BERce: Inmisiones dafínas en los ctultivos agricolas y de jardines. Investigación realizada por encargo de la Industrja Federal Alemana.

Investigaciones del año 1955 a 1958 (al aire libre), realizadas sobre trigo de jnviemo, centeno de invietno y colza de inviemo. (Ver texto.)

W. H. Fuchs: Investigación realjzada por encargo de la Unión de Fabricantes de Cemento Alemanes (W. D. Z.) (No bay publicación.)

Investigaciones patológicas previas sobre plantas estudiando las consecuentes influencias de la pulverización de las plantas. Ningún resultado.

F. Scheffer: Investigación encargada por la Unión de Fabricantes de Cemento Alemanes. Informes. Publicación prevista.

Resultados de ensayos celebrados al aire libre durante los años 1958 y 1959 . (Ver texto.)

\section{Influencia del polvo del horno de cemento sobre los animales}

Cohrs y Trautwean llevaron a cabo, en el Instituto Patológico de la Escuela de Veterinaria de Hannóver, amplias investigaciones sobre ganado vacuno, ovejas y conejos. Fl origen de estas investigaciones fue un informe que tuvo que realizar el Prof. Cohrs sobre Ios perjuicios causados a los verados del Parque Zoológico de Hannóver.

En un principio, las enfermedades de los venados fueron atribuidas a la caída de polvo cle las cercanas fábricas de cemento de Misburg. Sin embargo, se descubrió, en una inspección del lugar, que por las parcelas de los venados atravesaban canales de desagüe de unas construcciones de urbanización reciente. Eliminadas las cañerías desaparecieron también las enfermedades de los animales. Esto nos demuestra claramente con qué ligereza se atribuyen los trastornos patológicos a la influencia del polvo del horno de las fábricas de cemento. Podemos citar otros casos cuyas causas patológicas no son de conocimiento tan patente: tenemos, por ejemplo, el llamado «tétano de pastos», que. entre otras causas, tiene la de la falta de potasio. Otros son los casos de frigidez temporal del ganado vacuno, cuyas causas hay que buscar en la falta de manganeso de los piensos. Es digno de mención que, por ejemplo, en la región de Nordrheim-Westfalen, aproximadamente un 40 por 100 del ganado vacuno padece la enfermedad del distoma hepático. Todas ellas son enfermedades y causas patológicas que no tienen relación alguna con la inmisión de polvo, y las cuales pueden ser determiradas únicamente mediante cuidadosas investigaciones veterinarias.

El Prof. Cohrs comenzó una amplia serie de ensayos con 2 bueyes, 17 corderos y 16 conejos, a los cuales se había suministrado pienso con ciertas dosis de polvo del horno de cemento. En el primer ensayo, que tuvo lugar durante 60 días, entre el 12 de agosto y el I de noviembre de 1957 , se suministró a cada animal, por día, $2,5 \mathrm{~g}$ de polvo del horno de cemento.

En ninguno de los animales se apreció especie alguna de cuadro clínico ni durante la investigación ni después de su sacrificio.

En un segundo ensayo, que tuvo lugar del 4 al 27 de noviembre de 1957 , fueron suministradas a los animales dosis de polvo considerablemente incrementadas hasta $33 \mathrm{~g}$ por día y por animal. Este experimento fue aplicado, entre otros, a animales que ya habían sufrido la primera prueba. Tampoco durante este experimento no se apreció en animales ningún trastomo clínico. ni siquiera de pérdida de peso. 
Tampoco la introducción intranasal de grandes cantidades de polvo del horno de cemento durante un período de 4 a 9 meses, tuvo ninguna importante consecuencia perniciosa en el estado general de los animales, aunque se produjeron pequeños fenómenos catarrales en el aparato respiratorio y ligerísimas inmisiones de polvo en los pulmones.

Investigaciones patologoanatómicas e histológicas determinaron la no aparición de acciones fibrógenas del polvo del horno de cemento y demostraron que los órganos fisiológicos de defensa y de filtro son suficientes para limpiar el tejido pulmonar de, incluso, repetidas e incrementadas dosis de polvo.

También otras investigaciones confirmaron lo inofensivo del polvo del horno de cemento y la capacidad del organismo animal de soportar, sin consecuencias dañinas para la salud, incluso las determinadas dosis de polvo fuertemente aumentadas. Me refiero a la prueba realizada con 16 conejos a los que se les introdujo polvo por vía traqueal hasta los pulmones. Esta prueba tuvo un significado más bien teórico y puso de relieve las reacciones celulares, la absorción del polvo y, finalmente, la purificación de los pulmones.

Al final de sus exactas y amplias investigaciones llegó Cohrs a la conclusión final de que «el polvo del homo de cemento no tiene ninguna influencia especial sobre la salud, como causa originaria de enfermedades». Solamente cabría esperar daños cuando, aparte del polvo del horno de cemento, influyera sobre los animales otro producto tóxico, como, por ejemplo, el gas de flúor.

\section{Influencia del polvo del horno de cemento sobre las plantas}

Por encargo de la Unión de Fabricantes de Cemento Alemanes fueron llevados a cabo ciertos ensayos en el Centro de Investigaciones Biológicas del Carbono, Sociedad Registrada, de la localidad de Essen-Bredeney, bajo la dirección de los doctores Stratmann y van Haut. La Unión de Fabricantes de Cemento Alemanes tiene en su poder un amplio informe de tales investigaciones. Consisten, en primer lugar, en ensayos de laboratorio, los cuales fueron realizados sobre remolachas, patatas, alubias, pastos, avena y zanahorias, durante los años de 1954 a 1956.

Las primeras cantidades de polvo aplicadas fueron de 1 a $3 \mathrm{~g} / \mathrm{m}^{2} /$ día, sin que el control señalase ningún resuitado especial sobre las plantas. Por tanto, hubo que incrementar las cantidades de polvo a $6,12,24$ e incluso $48 \mathrm{~g} / \mathrm{m}^{2} /$ día, para ver si de esta manera se provocaba algún daño. A excepción de la avena, no se apreció ninguna influencia dañina sobre las plantas utilizadas. Ahora bien, Ia avena es conocida por susceptibilidad a la influencia de la sal, y representa, por esta razón, un objeto específico de investigación para ensayos de este tipo concreto (la influencia de la sal). Sin embargo, hay que tener en cuenta que los resultados obtenidos en los ensayos de laboratorio no pueden ser aplicados sir reservas a las pruebas que se verifiquen al aire libre. La tesis doctoral de $\mathbf{H}$. Fortmann, que trata ampliamente sobre la bibliografía, estudia, en su parte experimental, las investigaciones sobre cultivos en invernaderos. Ella está relacionada, en primer lugar, con los ensayos sobre empresas horti-floricultoras. Fortmann llegó a la conclusión de que con cantidades de polvo de $0,7 \mathrm{~g} / \mathrm{m}^{2} / \mathrm{día}^{\prime}$ la cobertura que se forma, de polvo, sobre las ventanas de los arriates del invernadero origina unas ligeras consecuencias dañinas sobre las plantas jóvenes, que puede demostrarse al comprobar una cierta disminución en la calidad y en la cantidad de los productos. Si se triplica la dosis de polvo, los cultivos en invernadero no resultarán comerciales.

Berge ha realizado, por encargo de la Industria Federal Alemana, unas investigaciones al aire libre sobre trigo de invierno, centeno de invierno y «colza» de invierno. Las cantidades de polvo utilizadas ascendieron a $1,5 \mathrm{~g} / \mathrm{m}^{2} /$ día. Las investigaciones se extendieron a lo largo de los años 1955 a 1958. Tampoco en este caso pudo ser demostrada estadísticamente la diferencia entre las parcelas cubiertas por el polvo y las no cubiertas.

Concretamente, en el cuarto año de ensayos (1958) aparecieron sobre el trigo de invierno ciertas enfermedades, en proporciones anormales. Pero tampoco en este caso fue posible demostrar estadisticamente las diferencias entre las parcelas cubiertas de polvo y las que no lo estaban. 
Los resultados de las anteriormente citadas investigaciones no fueron lo suficientemente amplios como para proporcionar una idea exacta sobre la influencia de las inmisiones de polvo en los alrededores de las fábricas de cemento. Sin embargo, esto aparecía siempre, y cada vez más, como una cuestión interesante, ya que incluso algunas fábricas de cemento se vieron complicadas en pleitos con sus vecinos agrieultores. Habían sido hechos unos informes por parte de los agricultores, durante el transcurso del pleito $y$ con motivo de éste. Estos informes demostraban una indudable influencia dañina del polvo del horno de cemento sobre los cultivos agrícolas. La Unión de Fabricantes de Cemento Alemanes realizó, a la vista de esto, con dos Institutos de la Universidad de Göttingen-el Instituto Patológico de Plantas y el Instituto Agroquímico-unos trabajos de investigación, que deberían servir para aclarar la influencia de la inmisión del polvo del horno de cemento.

En el Instituto Patológico de Plantas fue llevada a cabo en el año 1957 una serie de investigaciones para probar si la puiverización de distintas plantas, especialmente en estado de crecimiento, tiene alguna relación con la susceptibilidad al ataque de enfermedades y parásitos. Todas estas investigaciones discurrieron sin resultado, de tal manera que se ha prescindido de continuar los experimentos en el ámbito de este programa de investigaciones. Sin embargo, se ha mantenido el contacto con el Prof. Fuchs, para recibir rápida información sobre otras investigaciones experimentales de la cuestión de la influencia del polvo en los casos de enfermedades de plantas. Fue requerido, por ejemplo, sobre el ataque de la enfermedad, en el ensayo sobre la remolacha, llevado a cabo al aire libre en el año 1958 en el Centro Provincial del Fomento de la Explotación Agrícola (Dr. Fortmann. Bochum) en Menden. Este informe llevó al resuitado de que el ataque de la herrumbre de la remolacha no tiene ninguna relación con el polvo del homo de cemento. No existen diferencias estadísticamente demostradas entre las parcelas cubiertas de polvo y las no cubiertas, en este ensayo verificado en pleno campo.

\section{Ensayo celebrado en el campo, durante el curso 1958.59, en el Instituto Agroquímico de Göttingen}

Las investigaciones al aire libre, acordadas en un gran programa de ensayos, con el Prof. Scheffer, del Instituto Agroquímico, debían servir para aclarar las siguientes cuestiones:

1. Qué influencia tienen sobre el suelo las cantidades de polvo del horno de cenento depositadas en los alrededores de las fábricas de cemento (con respecto a las circunstancias biológicas y químicas).

2. En qué grado serán influenciados el crecimiento y el rendimiento de las plantas, desde su germinación hasta su madurez.

3. ¿Pueden ser atenuados o incluso eliminados los daños que se presenten, mediante medidas agrícolas?

Debido a circunstancias favorables tuvimos que enfrentarnos, en las campañas agrícolas de 1958 y 1959 , con dos condiciones climatológicas muy extremas, de tal manera que hay que dar un carácter universal a los resultados de los ensayos de ambos años.

Las figuras 1 y 2 muestran una perspectiva de la prueba celebrada al aire libre en los años 1958 y 1959 .

El lugar de este ensayo agrícola fue situado en la salida nordeste de Göttingen, sobre arcilla mojada de cerca de 1 in de espesor. Las semillas sembradas en años anteriores fueron:

1955: Trigo de inviemo y remolacha.

1956: Lino y remolacha.

1957: Centeno de verano y colza. 


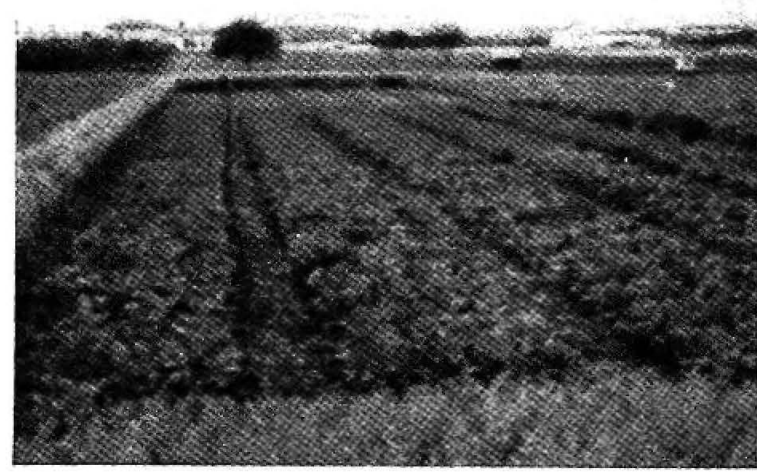

Fig. 1. Vista general del campo de ensayos (año 1958) tres meses después de la siembra.

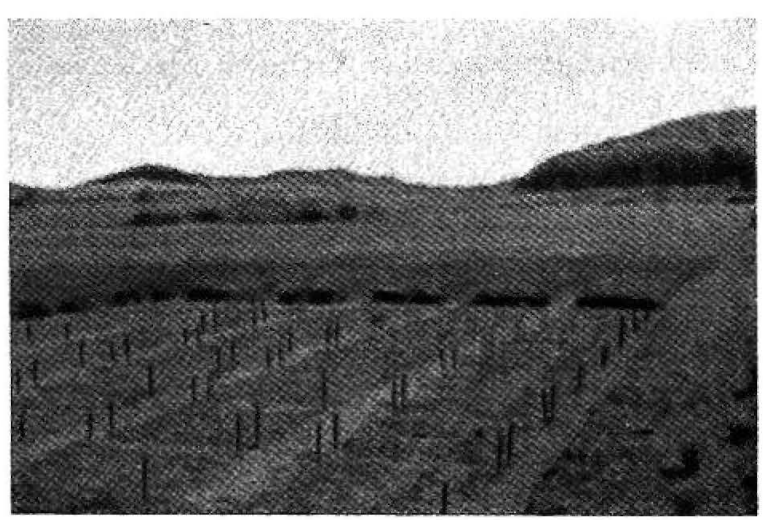

Fig. 2. Vista general del campo de ensayos (año 1959) dos meses después de la siembra.

La reacción del suelo antes de comenzar el ensayo de 1958 era: $\mathrm{pH}=5,8$. El contenido de $\mathrm{CaO}$ era prácticamente nulo. Además, fueron obtenidos: $39 \mathrm{mg}$ de $\mathrm{P}_{2} \mathrm{O}_{5}$ soluble en lactato por cada $100 \mathrm{~g}$ de suelo; y $19,5 \mathrm{mg}$ de $\mathrm{K}_{2} \mathrm{O}$ soluble en lactato de cada $100 \mathrm{~g}$ de suelo.

Para tomar en consideración también este efecto básico del polvo del horno de cemento del aire, en las investigaciones concernientes se hicieron ácidas la mitad de todas las parcelas antes de empezar la campaña del cultivo (para examinar de esta manera la influencia básica del polvo sobre el suelo), la otra mitad de las susodichas parcelas fue abonada de forma fisiológica alcalina (1).

En el primer año de ensayo estaban previstas para la siembra cuatro clases de frutos: avena, pastos, trébol rojo y remolacha.

Como mayor dosis de polvo fue escogida la de $1,5 \mathrm{~g} / \mathrm{m}^{2} /$ día, durante 10 días, la cual debía sobrepasar la de las situaciones más desfavorables de los alrededores de los núcleos de industrias de cemento. El período fue de 10 días para compensar las diferentes direcciones del viento que se dan en la realidad y, en consecuencia, las interrupciones en la caída de polvo; según esto, en determinadas parcelas se pulverizó durante 10 días con una dosis de $1,5 \mathrm{~g} / \mathrm{m}^{2} /$ día, y, a continuación, una pausa de 10 días. En otra serie de ensayos la dosis fue de $2,25 \mathrm{~g} / \mathrm{m}^{2} /$ día, durante 10 días y, seguidamente, una pausa de 20 días. Estas cantidades corresponden, por término medio, a una inmisión diaria de polvo de $0,75 \mathrm{~g} / \mathrm{m}^{2} /$ día, cantidad que se mantuvo como dosis diaria en una cuarta serie de pulverizaciones. Finalmente, se dejó para el final una última serie de pruebas sin aportación de polvo.

Resumiendo, se suministraron las siguientes dosis de polvo:

$$
0
$$

$0,75 \mathrm{~g} / \mathrm{m}^{2} /$ día

$1,50 \mathrm{~g} / \mathrm{m}^{2} /$ día

$1,50 \mathrm{~g} / \mathrm{m}^{2} /$ día; durante 10 días y finalizando con una pausa de 10 días.

(Esto corresponde a una media de $0,75 \mathrm{~g} / \mathrm{m}^{2} /$ día.)

En total, tenemos que tener en cuenta que cada fruto tiene 10 grupos de ensayos. Además, cada grupo de ensayos fue repetido cuatro veces, para poder asegurar de esta manera los resultados. Por tanto, se cuentan hasta 160 ensayos de parcelas que consisten en pruebas con avena, pastos, trébol rojo y remolacha.

(1) En el abono fisiológicamente ácido, el nitrógeno se encontraba como sulfato amónico y el fósforo como superfosfato. En el abono fisiológicamente básico, ambos elementos se presentaban como sulfato amónico cálcico y fosfato Thomas, respectivamente. En todos los casos, la potasa siempre fue una solución del 40 por 100 . 


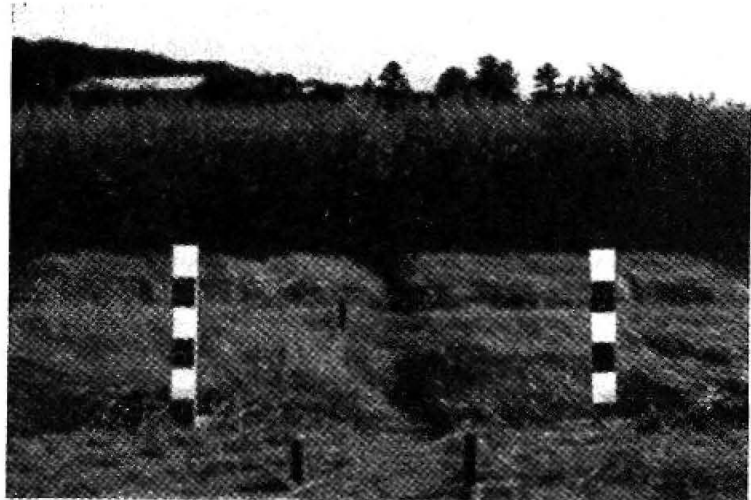

Fig. 3. Pasto (año 1958) antes del primer corte; a la izquierda, ninguna pulverización; a la derecha, $1,5 \mathrm{~g} / \mathrm{m}^{2} /$ día.

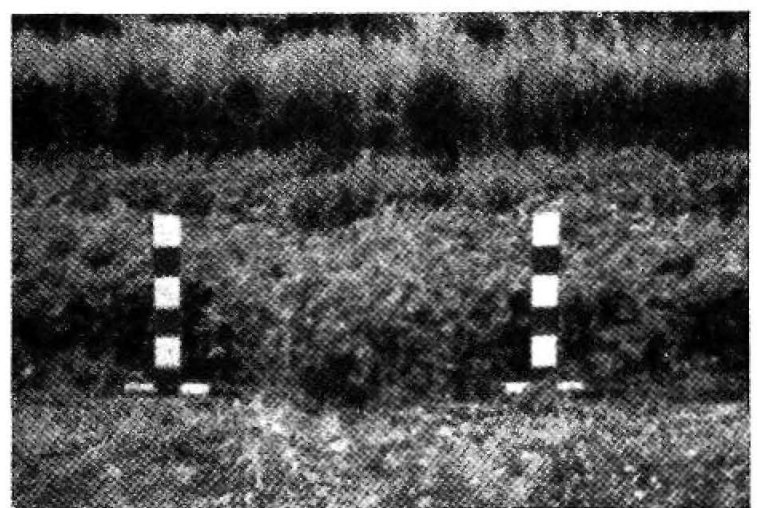

Fig. 4. Trébol rojo antes del primer corte: a la izquierda, ninguna pulverización; a la derecha, $1,5 \mathrm{~g} / \mathrm{m}^{2} /$ día.

Los ensayos se realizaban, cada uno, sobre una superficie de $4 \times 5=20 \mathrm{~m}^{2}$. Las parcelas fueron dispuestas de tal manera que se clasificaron según las dosis 'de polvo estadísticamente consideradas (2).

La totalidad del polvo del horno de cemento empleado en los ensayos procede de la purificación de un horno de vía húmeda provisto de sistema de despulverización eléctrica.

Contiene, entre otras sustancias: 29,3 por 100 de piedra caliza y 3,1 por 100 de $\mathrm{K}_{2} \mathrm{O}$. (La piedra caliza viene expresada como $\mathrm{CaO}$.)

Las condiciones atmosféricas a lo largo del ensayo del año 1958 habían influido sobre la siembra y la recolección del campo de pruebas de manera manifiesta. Una vez retrasada la siembra a causa del muy prolongado invierno (la primera siembra se celebró el 22 de abril de 1958), se destacó por otra parte un agosto con relativamente alta cantidad de lluvia en comparación con el término medio de los respectivos meses de años anteriores. El pasto y el trébol pudieron ser recogidos por segunda vez. La caída de polvo había comenzaao igualmente el 22 de abril de 1958 . El año 1958 puede ser considerado en general como un año húmedo.

Durante el período completo de crecimiento no fue hecha observación alguna que indicase que las plantas habían sido perjudicadas de alguna manera, ni en sentido positivo ni negativo. Teniendo en cuenta que el polvo diariamente caído no había sido arrastrado por las abundantes lluvias, a causa de la humedad, debería haber efectuado una acción corrosiva sobre las hojas, en caso de que realmente existiera tal acción dañina por parte del polvo respecto de las plantas. No fueron observadas en absoluto tales suposiciones.

Los pastos y el trébol fueron recogidos por primera vez a finales de julio de 1958. Las figuras 3 y 4 muestran el trébol y el pasto antes de la primera recolección $\mathrm{y}$, por cierto, aparecen paralelamente la parcela con mayor dosis de polvo $\left(1,5 \mathrm{~g} / \mathrm{m}^{2} /\right.$ día $)$ y la parcela que no había recibido polvo alguno. A mediados de septiembre fueron recolectados por segunda vez el pasto y el trébol.

Tanto en la primera recolección como en la segunda no fueron influenciadas desfavorablemente las cantidades recogidas. No se apreciaron más diferencias que las debidas a los errores mínimos necesarios de todo ensayo. Tampoco la clase de abono, fisiológicamente ácido o fisiológicamente básico, produjo acción alguna. Tampoco aparecen diferencias apreciables en los análisis de nitrógeno, ácido fosfórico y potasio (3).

(2) La realización exacta del ensayo se explicará en fecha próxima, con motivo de una publicación que realizará el profesor Scheffer.

(3) Los resultados numéricos de la cosecha ensayada, asi como los de todas las demás, se conocerán en el próximo artículo del profesor Scheffer. 

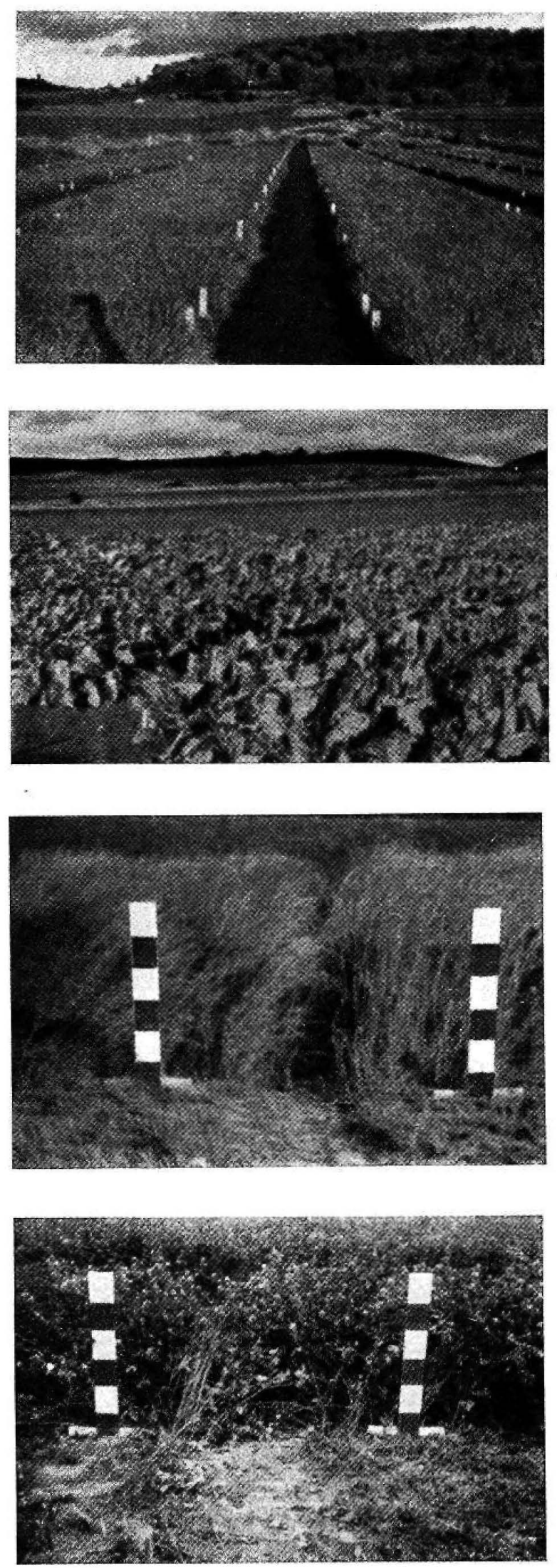

Fig. 5. Campo de ensayos (año 1958) antes de la recolección de remolacha, a mediados de octubre. Fig. 6. Vista parcial del campo de remolacha a mediados
de octubre de 1958 (la sección del grabado contiene parcelas con todos los tipos de pulverización).

Fig. 7. Pasto (año 1959) antes de su primer corte (segundo año de crecimiento): a la izquierda, ninguna pulverización; a la derecha, $1,5 \mathrm{~g} / \mathrm{m}^{2} /$ dia.
Fig. 8. Trêbol rojo (año 1959) antes de su primer corte (segundo año de crecimiento): a la izquierda, ninguna pul-
verización; a la derecha, $1,5 \mathrm{~g} / \mathrm{m}^{2} / \mathrm{dí}^{2}$. 
La avena fue recogida en los días 25 y 26 de agosto de 1958. Las cantidades de paja y trigo cosechadas en las parcelas pulverizadas son, por regla general, algo más bajas que en las no pulverizadas. En el cálculo de error no están demostradas estas diferencias como depresiones auténticas de la cosecha. Una comparación entre los abonos ácidos y los básicos, demuestra que en el caso de abonos fisiológicamente ácidos las cantidades de trigo son más altas y las de paja más inferiores. La combinación de abonos fisiológicamente ácidos con la pulverización nos ofrece una proporción más favorable de trigo y paja. Los análisis de nitrógeno, ácido fosfórico y potasio no señalan diferencia alguna imputable a la pulverización.

La recolección de la remolacha azucarera se realizó el 16 y el 18 de octubre de 1958 . La figura 5 proporciona una perspectiva del campo de remolacha poco antes de la recolección, es decir, cinco meses y medio después de la siembra. El pasto (en la parte anterior del grabado) se encontraba ya entonces tres semanas después del segundo corte. La figura 6 da una vista parcial de la remolacha. Los límites de las parcelas apenas pueden apreciarse a causa del crecimiento de las hojas. La sección que se observa contiene, sin embargo, parcelas con todos los tipos de pulverización. No son apreciables en la figura diferencias en el crecimiento de las hojas. Según demuestran los resultados de la recolección, la pulverización no alteró las cantidades recolectadas ni de azúcar ni de remolacha. Investigaciones realizadas sobre la remolacha no permiten apreciar ninguna infiuencia a causa del polvo. También el nitrógeno, el ácido fosfórico y el potasio permanecen invariables.

Estos fueron los resultados de los ensayos de 1958.

La pulverización fue continuada acto seguido de la recolección, a lo largo de todo el invierno; sin embargo, no se pulverizó a diario, sino que se empleó el polvo, en cantidades proporcionales, sólo cada 10 días. De esta manera se continuó hasta el comienzo de la siembra del ensayo de 1959.

En las pruebas del año 1959 se limitó el programa a investigaciones sobre remolacha azucarera, pasto y trébol rojo. La extraordinarłamente favorable primavera permitió la siembra algo antes que en el año anterior. Las remolachas pudieron sembrarse el 8 de abril de 1959. El pasto y el trébol rojo no fueron sembrados de nuevo, sino que se observó el crecimiento de las parcelas del año anterior, continuando con la pulverizaçión durante un segundo año de pruebas.

El año 1959 se caracterizó por una duradera sequía, la cual ha restultado desventajosa para la recolección en su segundo corte (pasto y trébol rojo).

El pasto fue recolectado una sola vez; esta vez fue el 22 de mayo de 1959 . Ni en el caso de haber utilizado el abono ácido ni cuando se utilizó el básico, fueron apreciadas diferencias en las cantidades recolectadas. La pulverización no llevó consigo ninguna influencia. En conjunto resuitaron mejores los abonos básicos; eran superiores a los ácidos. Nitrógeno, ácido fosfórico y potasio no sufrieron alteración alguna.

El trébol rojo fue cortado por primera vez el 10 de junio y por segunda vez el 31 de julio de 1959. Aquí resultan las cosechas de las parcelas pulverizadas claramente superiores a las de las no pulverizadas. Fueron especialmente significativas las diferencias habidas en el primer corte. También resultaron superiores los abonos ácidos en relación con los básicos. En el segundo corte estas características no aparecieron tan claras, debido a las grandes diferencias habidas (causadas por la sequía). También la recepción de nitrógeno fue favorablemente influida por la pulverización. El contenido de fósforo y potasio era igual en todas las parcelas de ensayos.

Las figuras 7, 8 y 9 representan de nuevo el pasto y el trébol rojo, respectivamente, antes de la primera recolección. Nuevamente están también aquí representadas las parcelas con las dosis de pulverización más elevadas $\left(1,5 \mathrm{~g} / \mathrm{m}^{2} /\right.$ día $\left.^{2}\right)$, al lado de parcelas no puiverizadas. 


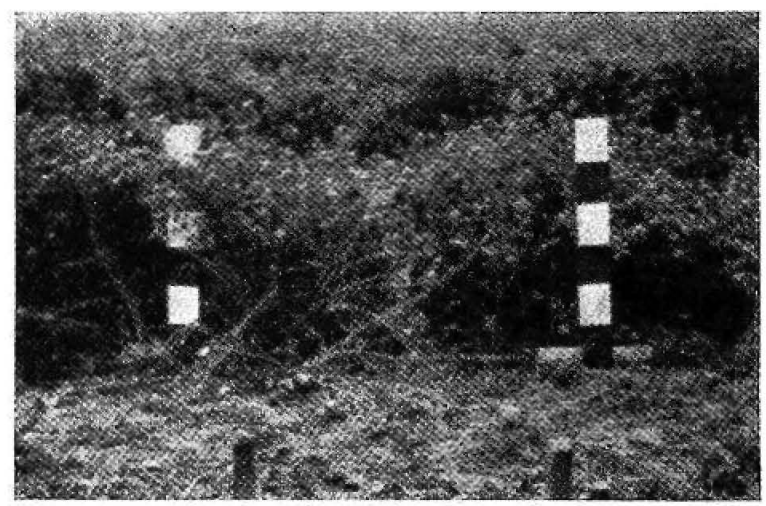

Fig. 9. Trébol rojo (año 1959) como el del grabado núm. 8: a la izquierda, $1,5 \mathrm{~g} / \mathrm{m}^{3} /$ día (10 dias de pulverización $y$

Además, aparece en la figura 9 una comparación entre una parcela no pulverizada y otra que recibió durante 10 días una dosis de $1,5 \mathrm{~g} / \mathrm{m}^{2} /$ día, finalizando en pausas de 10 días (dosis media de polvo de $0,75 \mathrm{~g} / \mathrm{m}^{2} /$ día). Esta figura demuestra que tampoco, mediante un sistema de pulverizaciones interrumpidas, aparece alteración alguna.

El 21 de octubre de 1959 fue recogida la remolacha. De un estudio comparativo de los resultados de la recolección resultó que a causa de la pulverización se incrementaron las cantidades recolectadas de remolacha. Esto resulta especialmente claro en el caso del abono fisiológicamente ácido. Sin embargo, el porcentaje de azúcar de la remolacha no sufrió influencia alguna. Lo cual hay que atribuir a la favorable influencia de la combinación: «polvo-abono ácido», y que no tiene una explicación satisfactoria y clara. Este fenómeno podría explicarse por el bajo contenido del nitrógeno dañino, lo cual implica mejores condiciones de crecimiento. El contenido de este pernicioso nitrógeno era más bajo en las parcelas pulverizadas.

Por el contrario, la cantidad de hojas era algo menor en el caso de las remolachas pulverizadas.

Asimismo, un contacto prolongado del polvo del horno de cemento con las hojas no ha ocasionado influencia alguna perniciosa. A causa de los meses de sequía, el polvo permaneció sobre las hojas de 3,5 a 4 meses. Si en realidad existiera una acción corrosiva por parte del polvo, ésta debería haber quedado -de manifiesto en el ensayo de este año, debido a la posibilidad de producirse

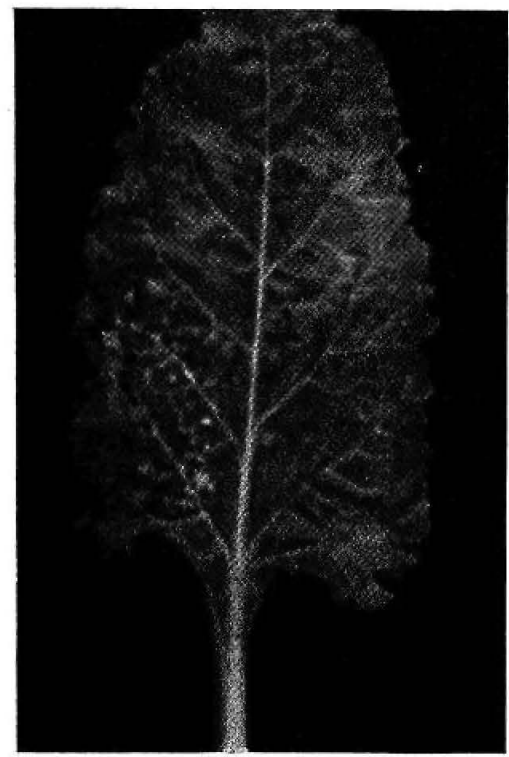
una continuada influencia sobre la superficie total de las hojas, a causa del rocío de las primeras horas de la mañana. Sin embargo, en ningún caso ha sido posible determinar influencia perniciosa alguna. La figura 10 muestra una hoja de remolacha, cuya mitad se ha limpiado de polvo. Sobre el lado izquierdo de la hoja se puede apreciar el polvo, el cual ha permanecido sobre la superficie de la hoja durante un trimestre. Se puede ver claramente que la superficie de la hoja ha permanecido totalmente sana.

El Prof. Scheffer, en su informe sobre este experimento, llegó a las siguientes conclusiones finales: 


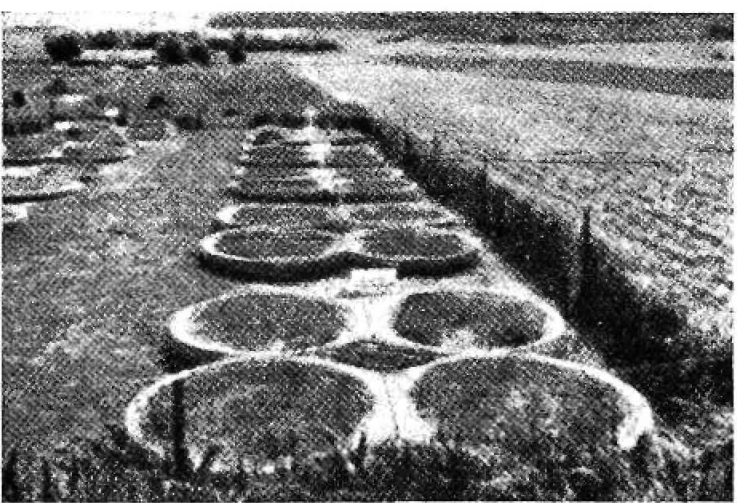

Fig. 11. Perspectiva del ensayo, sobre el suelo (año 1960), sin pulverización.

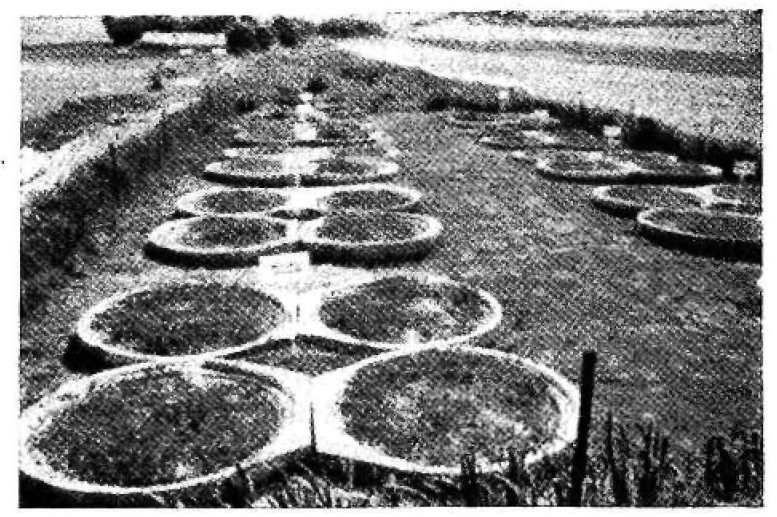

Fig. 12. Perspectiva del ensayo, sobre el suelo (año 1960), con $1,5 \mathrm{~g} / \mathrm{m}^{2} /$ día de pulverización.

1. Las precedentes investigaciones han demostrado que la sedimentación del polvo del horno de cemento no produce ninguna influencia inmediala sobre el crecimiento de las plantas.

2. Los frutos del ensayo, en los años que han durado las experiencias, han influido más o menos favorablemente sobre el suelo a causa del polvo del horno de cemento. El componente básico del polvo tuvo como consecuencia un incremento del valor del $\mathrm{pH}$ en el suelo.

3. Se recomienda contrarrestar, mediante medidas agrícolas preventivas, el exceso de cal, que eventualmente podría presentarse a causa de la sedimentación del polvo de cemento, especialmente en suelos ligeros con poca fuerza reguladora o tampón. Como tales medidas se recomienda: Incremento de la fuerza tampón del suelo, llevándolo a cabo mediante una adecuada sucesión de frutos (administración económica y beneficiosa del humus). Además de esto: abonos fisiológicos ácidos (superfosfato y sulfato de amonio). También se llama la atención sobre una posible solución secundaria: el cultivo de plantas no susceptibles a la acción de la cal.

Todavía no está solucionada definitivamente la cuestión que se basa en los ensayos de Göttingen (ver más arriba); es decir, la influencia sobre el suelo en sentido físico, químico y biológico. Sobre esto fueron planteados, en el año 1960, posteriores ensayos en Göttingen. Se investigó la medida en que son influidos los diferentes tipos de suelo (arcilla neutra, arcilla ácida, tierra arenosa, suelo originado por el desgaste del suelo debido a circunstancias atmosféricas y arena diluvial) mediante dosis constantes de $1,5 \mathrm{~g} / \mathrm{m}^{2} /$ día, de polvo del horno de cemento. Los diferentes tipos de suelo fueron introducidos en grandes tubos de hormigón, de $1 \mathrm{~m}$ de largo y $1,15 \mathrm{~m}$ de diámetro( correspondientes a $1 \mathrm{~m}^{2}$ de superficie del suelo). Los tubos fueron colocados en el suelo. Mediante estas medidas debían ser eliminadas las influencias eventuales de los tipos de suelo contiguos, dentro del campo de ensayos; por lo demás, se procedió lo más posible en concordanci乏 con las circunstancias naturales.

Las figuras 11 y 12 muestran que los tubos colocados en el suelo están distribuidos en grupos de a cuatro y los tubos de un mismo grupo tenían un mismo tipo de suelo. En el año 1960 fue sembrado trébol como primer fruto. Todavía no tenemos resultados definitivos. El ensayo deberá ser observado durante varios años.

\section{Acción del polvo del horno de cemento como abono}

También puede ser interesante tomar en consideración la acción del polvo del horno de cemento como abono potásico y cálcico, en relación con las observaciones sobre los efectos que fomentan el crecimiento mediante la inmisión de polvo del horno de cemento. 
El contenido de potasio del polvo del horno de cemento no es constante, sino que puede tener valores más o meroos altos de $\mathrm{K}_{2} \mathrm{O}$, en forma de sulfato potásico. Ya hace cuarenta años llamó la atención E. Schott, en una conferencia pronunciada en la Junta General núm. 43 de la Unión Alemana de Fabricantes de Cemento Portland, sobre el hecho de que el polvo de cemento recogido a la cabeza de un horno giratorio puede servir, en ciertas circunstancias, como abono combinado de potasio y cal. El polvo de entonces contenía mayor cantidad de $\mathrm{K}_{2} \mathrm{O}$ (cerca de un 20 por 100) y aproximadamente un tercio de $\mathrm{CaO}$ (cerca de un 10 111 por 100) de nuestro polvo de hoy. Con tal clase de polvo pudieron ser obtenidos ciertos éxitos como abono sobre pastos, patatas e, inciuso, plantaciones de trigo.

También en los tiempos modernos (1957) han sido realizados ensayos con polvo procedente de la purificación de gases mediante el sistema eléctrico, en los que se podía comprobar los efectos del polvo como abono sobre la avena y las patatas, tanto en ensayos de laboratorio como al aire libre. Estos ensayos dieron resultados positivos. Sin embargo, hay que tener en cuenta que los yacimientos alemanes, ricos en potasio, no dejan progresar comercialmente al polvo del horno de cemento como abono.

\section{Influencia sobre los cultivos forestales}

Una cuestión posterior, que exige especial atención, es la de la influencia del polvo del horno de cemento sobre la ciencia forestal.

Sobre este tema fue confeccionado, en el año 1956, en la Escuela Forestal de Tharandt (TH Dresden), y por G. Wolf, un trabajo diplomado titulado «Estudios sobre los daños que ocasiona el aire pulverizado sobre los bosques de las cercanías de las fábricas de cemento, deducidos de las investigaciones realizadas en Göschwitz (Saale) y Bad Berka.

Wolf determinó en este trabajo que sus investigaciones, celebradas de julio de 1955 a enero de 1956, habían arrojado auténticas emisiones dañinas, especialmente sobre coníferas, en los alrededores de las fábricas de cemento de Göschwitz y Bad Berka.

Ahora bien, hay que tener en cuenta que:

1. En la región de Göschwitz, además de la fábrica de cemento, emitían gases de escape en el ambiente una fábrica de vidrio $y$ otra de productos farmacéuticos. A esto ha aludido Wolf en su trabajo y con ello ha limitado sus consecuencias finales fundamentalmente.

2. Wolf escribe, más adelante, que las conf́feras investigadas están, en parte, sobre suelos muy pobres y, en consecuencia, muy susceptibles a los perjuicios ocasionables por la inmisión. Esto acontece, sobre todo, a una serie de pinos de Flandes investigados. Es digno de mención que junto a los pinos de Flandes dañados hay, sin embargo, algunos completamente sanos y que no muestran influencia dañina alguna.

3. La fábrica de cemento de Bad Berka es un simple molino de cemento. Sin embargo, las despulverizaciones de los molinos parecen ser incompletas; por ello Wolf menciona una expulsión de polvo de $3,5 \mathrm{t} /$ día. Aquí se trata del genuino polvo de cemento del horno. La escoria procedente de los altos hornos contiene, sin embargo, sulfuro cálcico, el cual es considerado especialmente tóxico para los cultivos foresiaies.

La cuestión de la influencia dañina del polvo del horno de cemento existente en el aire, sobre los árboles coníferos y de follaje, parece no estar suficientemente aclarada $\mathrm{y}$, por tanto, se intentan llevar a cabo posteriores investigaciones experimentales sobre este tema. 